\title{
INELASTIC NEUTRON SCATTERING STUDY OF FERROELECTRIC PHASE TRANSITION IN LITHIUM HEPTAGERMANATE $\left(\mathrm{Li}_{2} \mathrm{Ge}_{7} \mathrm{O}_{15}\right)$
}

\author{
Mitsuo W. Takeda ${ }^{1}$, Yukio Noda ${ }^{2}$, and Toshihisa Yamaguchi ${ }^{3}$
}

${ }^{1}$ Department of Physics, Faculty of Science, Shinshu University, Matsumoto 390-8621, Japan

${ }^{2}$ Institute of Multidisciplinary Research for Advanced Materials, Tohoku University 2-1-1 Katahira, Aoba-ku, Sendai 980-8577, Japan

${ }^{3}$ School of Science and Engineering, Meisei University, Hodokubo, Hino, Tokyo 191-8506, Japan

Keywords: Ferroelectrics, Soft phonon mode, Critical slowing-down, Inelastic neutron scattering, $\mathrm{Li}_{2} \mathrm{Ge}_{7} \mathrm{O}_{15}$

\section{Synopsis}

Phonon dispersions in ferroelectric lithium heptagermanate $\mathrm{Li}_{2} \mathrm{Ge}_{7} \mathrm{O}_{15}$ (LGO) were measured by inelastic neutron scattering in the vicinity of the transition temperature $T_{\mathrm{C}}=$ $283.5 \mathrm{~K}$. The (300) reflections show more pronounced temperature dependence than the $(500)$ reflections. The energy of a peak corresponding to the Brillouin zone-center phonon, around $0.5 \mathrm{meV}$ at $297.0 \mathrm{~K}$, decreases as the temperature decreases. Then, this inelastic component disappears into the central component at $288.0 \mathrm{~K}$, just above $T_{\mathrm{C}}$. The present phonon shows the typical soft mode feature and shows good accordance with the $B_{1 \mathrm{u}}$ mode observed by hyper-Raman and FTIR measurements. It is confirmed that the ferroelectric phase transition in LGO is associated with the soft optical phonon at the zone center. Moreover, elastic diffuse scattering is observed below $0.1 \mathrm{meV}$. This implies that there exists a critical phenomenon at energies below than $0.1 \mathrm{meV}$.

\section{Introduction}

Structural phase transitions can be classified into two types: (i) displacive-type transitions and (ii) order-disorder-type transitions. From the viewpoint of the soft mode concept proposed by Cochran, ${ }^{1)}$ the former is associated with a cooperative resonant mode (phonon), where a decrease in the phonon frequency should be observed. On the other hand, the latter is associated with a cooperative relaxation mode (Debye-like mode), where the divergence of relaxation time, i.e., critical slowing down, should be observed.

Some ferroelectrics have attracted considerable interest because of their peculiar behavior of exhibiting critical slowing down as well as the soft phonon mode during the phase transition. In order to interpret the dynamics of this type of phase transition, two models have been proposed. In the first model, only the soft phonon mode exists. As the transition temperature $\left(T_{\mathrm{C}}\right)$ is approached, the damping constant increases and the phonon frequency decreases. Consequently, the soft phonon mode becomes "overdamped," and in the vicinity of 
$T_{\mathrm{C}}$, it changes to the so-called "pseudo-relaxation mode." Apparently, it is difficult to distinguish between the pseudo-relaxation mode and the proper overdamped soft phonon mode.

In the second model, the relaxation mode and the soft phonon mode exist simultaneously. In regions rather far from $T_{\mathrm{C}}$, the phonon frequency decreases as $T_{\mathrm{C}}$ is approached. In the vicinity of $T_{\mathrm{C}}$, due to the coupling effect of the soft phonon, the relaxation mode changes to the soft mode and the relaxation time diverges.

It is found that lithium heptagermanate $\mathrm{Li}_{2} \mathrm{Ge}_{7} \mathrm{O}_{15}$ (LGO) undergoes a ferroelectric phase transition similar to that mentioned above. The purpose of this study is to determine the point in the Brillouin zone at which the polar phonon softens. In the present paper, the dispersion relation of the soft phonon mode and a critical phenomenon associated with low-lying energy levels will be discussed on the basis of the results of inelastic neutron scattering.

\section{Ferroelectric Phase Transition in LGO}

LGO undergoes a ferroelectric phase transition at $T_{\mathrm{C}}=283.5 \mathrm{~K}$. The space group of the paraelectric phase is $\mathrm{Pbcn}$ and that of the ferroelectric phase is $P b c 2_{1}$ with spontaneous polarization along the $c$-axis. ${ }^{2 \sim 4)}$ The size of the unit cell does not change during the transition $(Z=4)$. The crystal structure consists of strongly puckered layers of $\mathrm{GeO}_{4}$ tetrahedra linked by $\mathrm{GeO}_{6}$ octahedral, forming a three dimensionally bridged framework. The Li atoms occupy the vacant channels extending three dimensionally. ${ }^{3)}$

The dielectric constant along the $c$-axis shows a sharp increase around $T_{\mathrm{C}}$. The Curie-Weiss law holds only in a narrow temperature region of $\left(T_{\mathrm{C}}+4\right) \mathrm{K}$ with a small Curie constant $C=$ $4.6 \mathrm{~K}$. Below $T_{\mathrm{C}}$, typical $D-E$ hysteresis loops have been observed. ${ }^{4}$ The spontaneous polarization and coercive field estimated from the hysteresis loop are $0.02 \mathrm{mC} / \mathrm{cm}^{2}$ and 2.6 $\mathrm{kV} / \mathrm{cm}$ at $276 \mathrm{~K}$, respectively. The small Curie constant suggests an order-disorder-type transition. The specific heat has been measured by using AC calorimetry and shows small anomaly around $T_{\mathrm{C}}$. This suggests that the ferroelectric phase transition of LGO is the displacive -type one. ${ }^{5)}$

The softening of the optical phonons belonging to the $B_{1 \mathrm{u}}$ representation in the paraelectric phase has been confirmed by IR measurements, and the existence of the totally symmetric $A_{1}$ mode in the ferroelectric phase has been confirmed by both Raman and IR measurements. ${ }^{4}$ ${ }^{6 \sim 14)}$ Further, dielectric critical slowing down has been confirmed by dielectric dispersion measurements. ${ }^{15 \sim 17)}$ As mentioned above, two models have been proposed for this type of transition. In the first model, only the soft phonon mode exists. As $T_{\mathrm{C}}$ is approached, the soft phonon mode becomes overdamped, and in the vicinity of $T_{\mathrm{C}}$, it changes to the pseudo-relaxation mode. In the second model, the relaxation mode and the soft phonon exist simultaneously. In regions very close to $T_{\mathrm{C}}$, the soft mode characteristics of the phonon are transformed to the relaxation mode. 


\section{Experimental Procedure}

\section{3-1 Crystal growth}

Single crystals of LGO were grown in an ambient atmosphere by the Czochralski method from a stoichiometric melt at approximately $1300 \mathrm{~K}$ by employing a resistance furnace. Raw materials for the crystal growth were synthesized from the stoichiometric mixture of powdered $\mathrm{Li}_{2} \mathrm{CO}_{3}$ and $\mathrm{GeO}_{2}$ of $99.999 \%$ purity without $\mathrm{Li}$ isotopes by repeating solid-state reactions 3 times at $1123 \mathrm{~K}$ for 20 hours. The grown crystals were colorless and fully transparent and had clear natural growth surfaces as shown in Fig. 1.

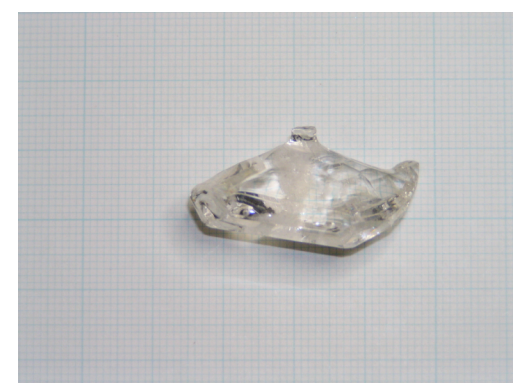

Fig. 1 Single crystal of LGO grown by Czochralski method.

\section{3-2 Inelastic neutron scattering}

For the neutron experiment, a single crystal was cut and mounted on an aluminum holder. The sample of LGO crystal had a rectangular parallelepiped shape with dimensions $15 \times 20 \times$ $18 \mathrm{~mm}^{3}$. Since the absorption coefficient of the crystal for thermal neutrons is negligibly small, no further shape refining was attempted. Intensity data of the Bragg reflection were collected by using the $\omega$-scanning mode. To investigate the mechanism of phase transition, the detailed temperature dependence of the dispersion curves of low-lying phonons around $T_{\mathrm{C}}$ was measured by the inelastic neutron scattering. The temperature of the sample was controlled by using a He-gas refrigerator type cryostat, which could be attached to the triple-axis spectrometers $4 \mathrm{G}$ and $\mathrm{C} 1-1$ in JRR-3M at Tokai.

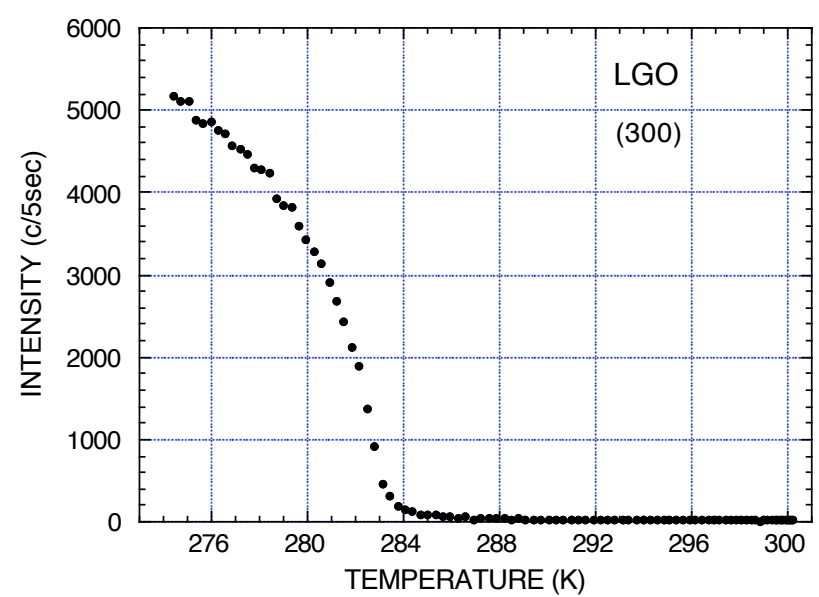

Fig. 2 Temperature dependence of (300) reflections of LGO. 


\section{Results}

The detailed temperature dependence of both elastic and inelastic components of the (300) and (500) reflections was measured. The (300) and (500) Bragg reflections appeared abruptly below $T_{\mathrm{C}}$ due to the change of the symmetry, and integrated intensity of (300) reflection is shown in Fig. 2. The (300) reflections show more pronounced temperature dependence than the (500) reflections. Typical profiles at (300) are shown in Fig. 3. The energy of a peak corresponding to the zone-center phonon, around $0.5 \mathrm{meV}$ at $297.0 \mathrm{~K}$, decreases as the temperature decreases. Then, this inelastic component disappears into the central component at $288.0 \mathrm{~K}$, just above $T_{\mathrm{C}}$. The present phonon shows the typical soft mode feature and shows good accordance with the $B_{1 \mathrm{u}}$ mode observed by hyper-Raman ${ }^{21)}$, Fourie transform infrared $(\text { FTIR })^{6,13)}$, and the backward wave oscillator tube $(\mathrm{BWO})^{9)}$ measurements.
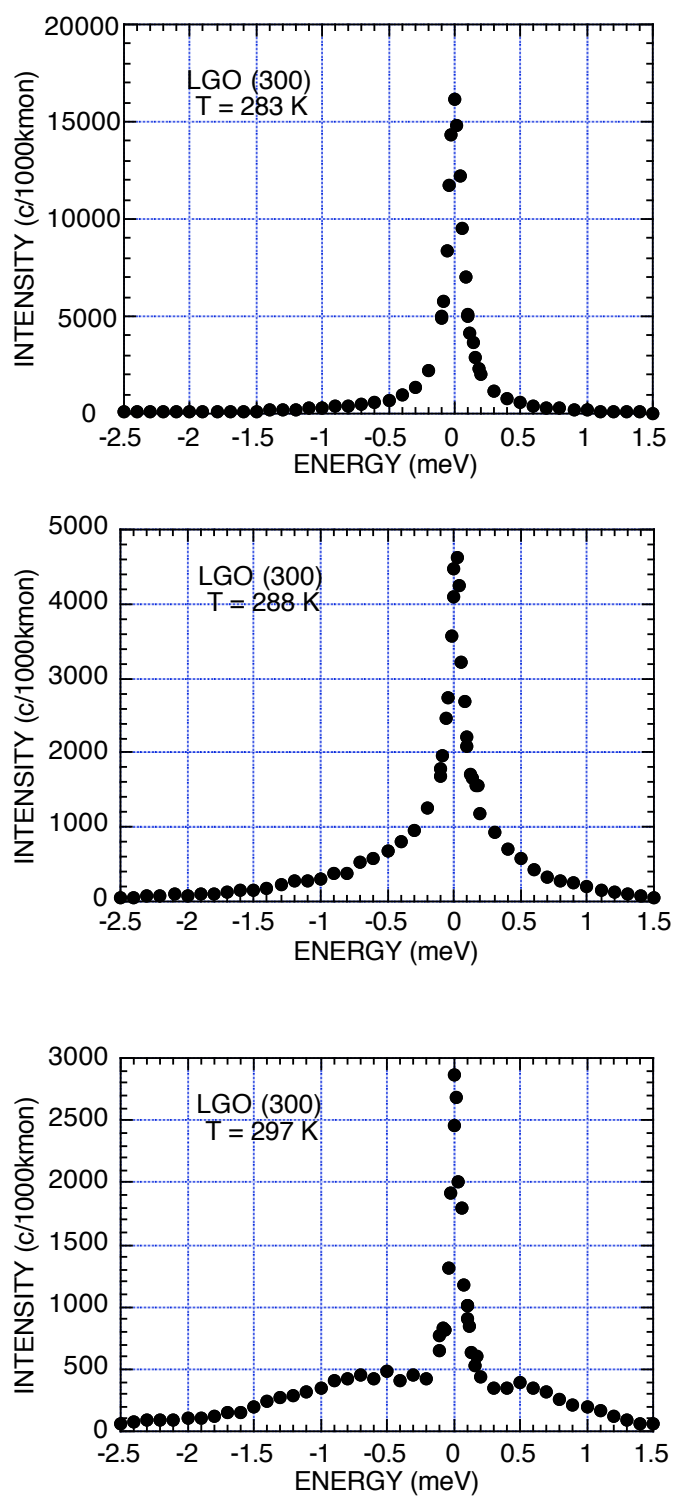

Fig. 3 Temperature dependence of inelastic peaks around (300) reflections of LGO. 
The dispersion curves of the lowest-lying phonon branch along the $a^{*}$ and $c^{*}$ directions are clearly observed to lie around the Brillouin zone center and showed the softening as $T_{\mathrm{C}}$ is approached (Fig. 4). Thus, it is confirmed that the ferroelectric phase transition in LGO is associated with the soft optical phonon at the zone center $(\Gamma$ point $)$.
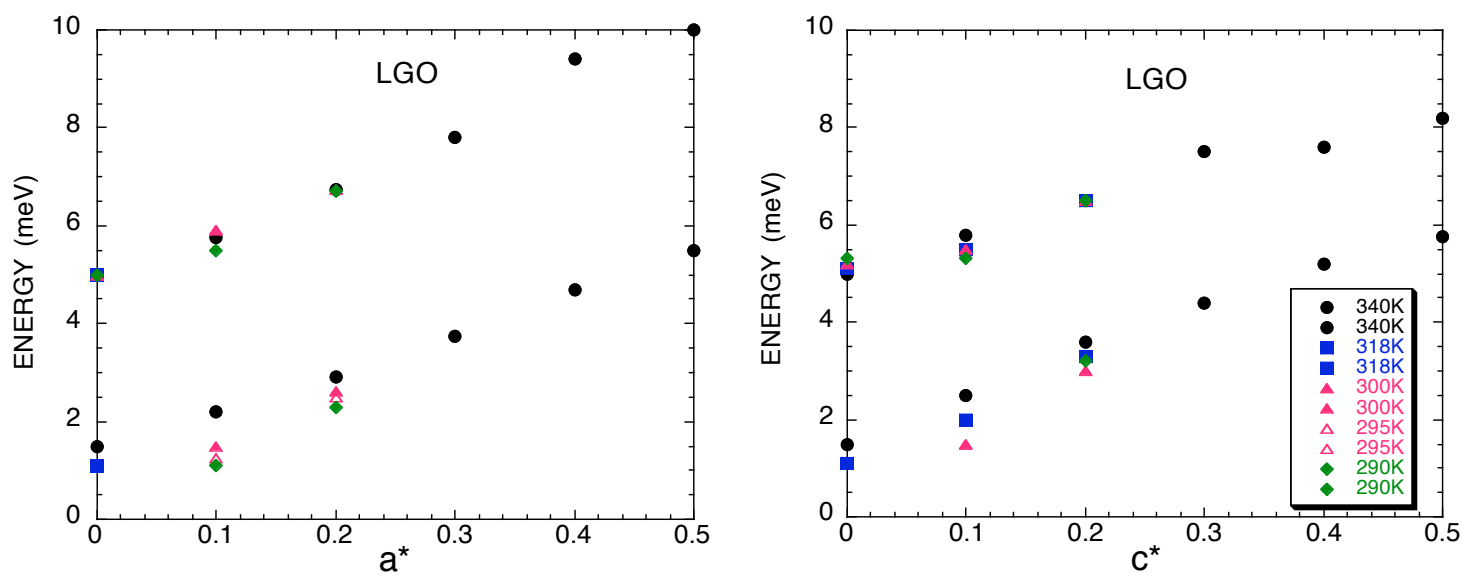

Fig. 4 Temperature dependence of dispersion curves of lowest-lying phonon branch along $a^{*}$ and $c^{*}$ directions

All the results obtained by $\operatorname{Raman}^{4)}$ and hyper-Raman scattering ${ }^{21)}, \mathrm{FTIR}^{6,13)}, \mathrm{BWO}^{9)}$, and inelastic neutron scattering (present study) are summarized in Fig. 5.

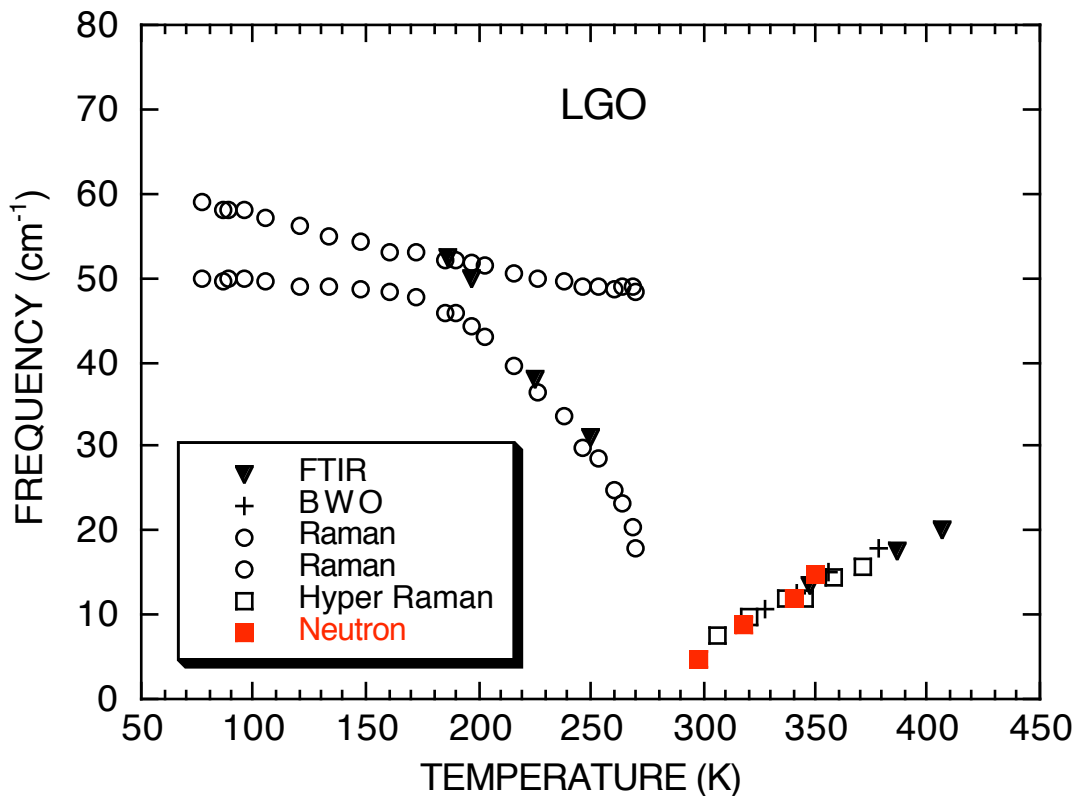

Fig. 5 Temperature dependence of soft phonon mode frequency. 
Moreover, elastic diffuse scattering is observed below $0.1 \mathrm{meV}$. As shown in Fig. 6, the intensity at (300) diverges at $T_{\mathrm{C}}$. The intensity at (3 $\left.0-0.04\right)$, slightly off from (300) reciprocal point, is also shown in the figure. This implies that there exists a critical phenomenon at energies below $0.1 \mathrm{meV}$.

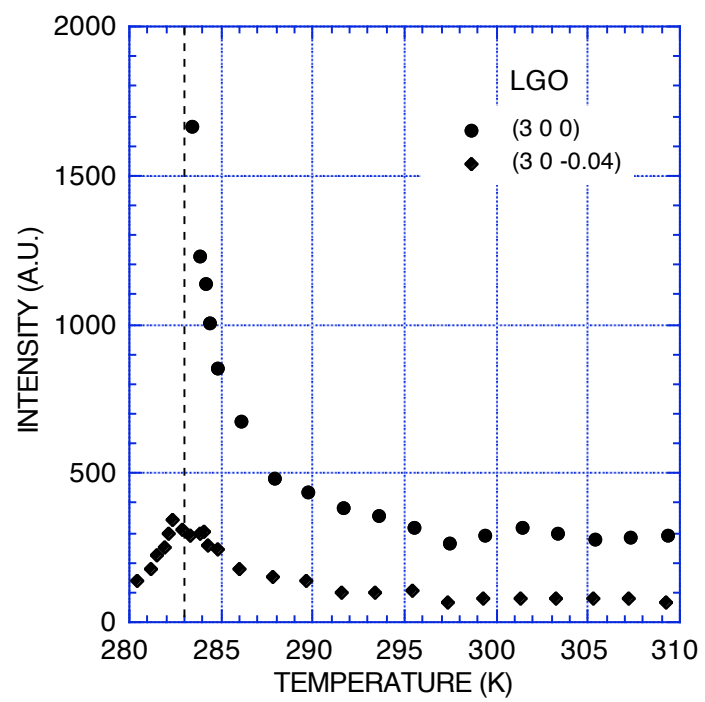

Fig. 6 Temperature dependence of (300) and (3 $0-0.04)$ reflections.

\section{Discussions}

From the crystal structure analyses by neutron diffraction carried out by Iwata et al. ${ }^{18,19)}$, it is concluded that the change within a framework composed of corner-sharing $\mathrm{GeO}_{4}$ and $\mathrm{GeO}_{6}$ polyhedra can be ascribed to the combined tilting motion of the tetrahedra, and the Li atoms in broad channels show a marked anisotropic spatial distribution along the channel direction in the paraelectric phase. The change in the framework of the polyhedra is interpreted as the result of the displacive-type transition freezing the tilting motion of the tetrahedra. This corresponds to the soft phonon mode observed in spectroscopic studies. The behavior of the Li atoms during the transition may be interpreted by using the split-atom model (order-disorder model), corresponding to the relaxation mode.

In the present study the existence of the $B_{1 \mathrm{u}}$ soft phonon mode at Brillouin zone center and the critical phenomenon corresponding to the relaxation mode are confirmed. Thus, the ferroelectric phase transition in LGO is associated with the polar soft phonon mode in the $B_{1 \mathrm{u}}$ representation at the zone center, and in the vicinity of the transition point, the soft mode characteristics are "transferred" from the $B_{1 \mathrm{u}}$ soft phonon mode to the relaxation mode with the same symmetry. Thus, the relaxation mode plays an essential role in the transition phenomenon. The transfer of the soft mode characteristics is estimated to occur in the frequency region around $1 \mathrm{~cm}^{-1}$. The simultaneous observation of the soft phonon mode and the relaxation mode must be quite important. 
Acknowledgements

The authors wish to thank Y. Iwata for the discussion on neutron measurements and $\mathrm{M}$. Horioka for the fruitful discussions on dielectric critical slowing down. They also wish to thank A. Sawada and Y. Ishibashi for the discussions on the transfer phenomena of the soft mode characteristics during structural phase transitions. The measurements were performed by using the neutron spectrometers $4 \mathrm{G}$ and $\mathrm{C} 1-1$ in JRR-3M at Tokai.

\section{References}

1. W. Cochran, Adv. Phys. (GB), 9 (1969) 387.

2. S. Haussuhl, F. Wallrafen, K. Recker, and J. Eckstein, Z. Kristallogr. (Germany), 153 (1980) 329.

3. H. Vollenkle, A. Wittman, and H. Nowotny, Monatsh Chem (Austria), 101 (1970) 46.

4. M. Wada, A. Sawada, and Y. Ishibashi, J. Phys. Soc. Jpn., 50 (1981) 1811.

5. K. Morikawa, T. Atake, M. Wada, and T. Yamaguchi, J. Phys. Soc. Jpn., 67 (1998) 1994.

6. M. Wada and Y. Ishibashi, J. Phys. Soc. Jpn., 52 (1983) 193.

7. H. Terauchi, S. Iida, Y. Nishihata, M. Wada, A. Sawada, and Y. Ishibashi, J. Phys. Soc. Jpn., 52 (1983) 2312.

8. M. Wada, A. Sawada, and Y. Ishibashi, J. Phys. Soc. Jpn., 53 (1984) 3319.

9. A. A. Volkov, G. V. Kozlov, Yu G. Gondharov, M. Wada, A. Sawada, and Y. Ishibashi, J. Phys. Soc. Jpn., 54 (1985) 818.

10. H. Orihara, M. Wada, and Y. Ishibashi, J. Phys. Soc. Jpn., 52 (1983) 1478.

11. A. Sawada, M. Wada, K. Fujita, and H. Toibana, Jpn. J. Appl. Phys., 24 (1985) Suppl. 24-2, 534.

12. M. Wada, K. Fujita, A. Sawada, and Y. Ishibashi, Jpn. J. Appl. Phys., 24 (1985) Suppl. 24-2, 488.

13. M. Wada, Ind. J. Pure \& Appl. Phys., 26 (1988) 68.

14. Y. Morioka, M. Wada, and A. Sawada, J. Phys. Soc. Jpn., 57 (1988) 3198.

15. M. Horioka, A. Sawada, and M. Wada, Ferroelectrics, 95 (1989) 233.

16. M. Horioka, A. Sawada, and M. Wada, J. Phys. Soc. Jpn., 58 (1989) 3793.

17. H. Yanagihara, M. Horioka, S. Furuta, and T. Yamaguchi, J. Phys. Soc. Jpn., 65 (1996) 1099.

18. Y. Iwata, I. Shibuya, M. Wada, A, Sawada, and Y. Ishibashi, Jpn. J. Appl. Phys., 24 (1985) Suppl. 24-2, 597.

19. Y. Iwata, I. Shibuya, M. Wada, A, Sawada, and Y. Ishibashi, J. Phys. Soc. Jpn., 56 (1987) 2420.

20. K. Krec, W. Steiner, and M. Wada, Acta Cryst., A49 (1993) 198.

21. M. Arai, M. Arima, A. Sakai, M. Wada, A, Sawada, and T. Yagi, J. Phys. Soc. Jpn., 56 (1987) 3213. 